\title{
An Experimental Study of Astigmatism Optical Correction on Visual Acuity Changes when being Corrected with Spherocylindrical and Spherical Equivalent Spectacle Lenses
}

\author{
Innocent Thawe* \\ Master's Degree in Optometry and Vision Therapy, Zomba Central Hospital, Malawi
}

Submission: January 12, 2019; Published: February 05, 2019

*Corresponding author: Innocent Thawe, School of Advanced Education, Research and Accreditation, Master's Degree in Optometry and Vision Therapy, Zomba Central Hospital, Zomba, Malawi

\begin{abstract}
Objective: To compare astigmatism optical correction on visual acuity changes when being corrected with spherocylindrical and spherical equivalent spectacle lenses.
\end{abstract}

Methods: A trial case, trial frame and log MAR visual acuity chart was used to determine corresponding power using a subjective refraction. 70 subjects (ages 15-22) were enrolled in the study. The cylindrical and spherical refractive outcomes were binocularly balanced among subjects during refraction sessions. The different visual outcome measures were obtained as follows:
a) Presenting visual acuity outcomes,
b) Visual acuity corrected by spherocylindrical lenses and
c) visual acuity corrected with spherical equivalent.

Results: The visual acuity obtained by spherocylindrical correction improved in a cylindrical power correction of all ranges well. The spherical equivalent correction visual acuity was decreasing as the cylindrical power was increasing.

Conclusion: Spherocylindrical correction is applicable in all cylindrical powers while spherical equivalent is applicable in small cylindrical powers. The spherical equivalent spectacle lenses that will give high quality results should be used in a cylindrical power of less than 1.00D.

Keywords: Visual acuity; Spherocylindrical; Cylindrical powers; Hyperopic; Human eye; Eyeball; Emmetropia; Astigmatism cornea; Monozygotic; Myopic; Dizygotic twins; Hyaloid fossa; Lens; Keratoconus; Trauma

Abbreviations: ATR: Against the Rule; BCVA: Best Corrected Visual Acuity; COLC: Circle of least confusion; IOL: Intraocular lens; WTR: With the Rule; SE: Spherical Equivalent; SAERA: School of Advanced Education, Research and Accreditation; SICS: Small incision cataract surgery; H/V: Horizontal / Vertical; ISNT: Inferior Superior Nasal Temporal; Log MAR: Logarithmic Minimum Angle of Resolution

\section{Introduction}

At birth, the human eye is hyperopic. As the child grows, the eyeball compensates for the hyperopia (farsightedness). When compensation is done properly it is known as emmetropia. The process of eye that is well coordinated that enables an image at far to fall on retina is known as emmetropization. When emmetropization has failed to take place properly it leads to refractive errors as explained (Filtcroft 2014). One of them is astigmatism or an eye with either egg shaped cornea or crystalline lens which causes light to be focused on two separate focal points. The irregularity of the front surface curve causes image distortions. Therefore, the most common two types of astigmatism cornea and lenticular astigmatism (Figure 1).
Goldschimdt contributed an opposing theory with extensive evidence that refractive error could be a hereditary dependent issue (Goldschimdt 2014). In this concept siblings' similarities were observed while parents and their off springs were not included in the literature. Also concurred in the study of same sex monozygotic twins in which it was found that $90 \%$ were within +/- 1.25D and 95\% within each other in +/- 1.65D of each other while dizygotic twin pairs only $52 \%$ were within +/- 1.25D within each other [1]. In similar setup of subjects of monozygotic and dizygotic twins as subjects, a sophisticated data collection was done using Humphreys 670 auto refractor to measure spherical equivalent, corneal astigmatism and total astigmatism concordance rate. It gave a concrete proof that 
myopic and hyperopic astigmatism were dominantly inherited refractive errors. There was a total variance of $47 \%$ to $49 \%$ in total astigmatism and $61 \%$ in cornea astigmatism with $95 \%$ confidence interval in the study [2]. In further substantiation on genes and environmental factors influence on refractive status a group of elderly monozygotic and dizygotic twins age around 63 to 76 years born between 1924 to 1937 were studied. It was concluded intraclass correlation of spherical equivalent of monozygotic twins was higher than of dizygotic twins [3]. The cornea was observed to have more influence on astigmatism because it accounts for $2 / 3$ of the total eye's refractive power. According to literature anterior surface is known to be neutralized by back surface's contribution (Bennet, Rabbert 1984).

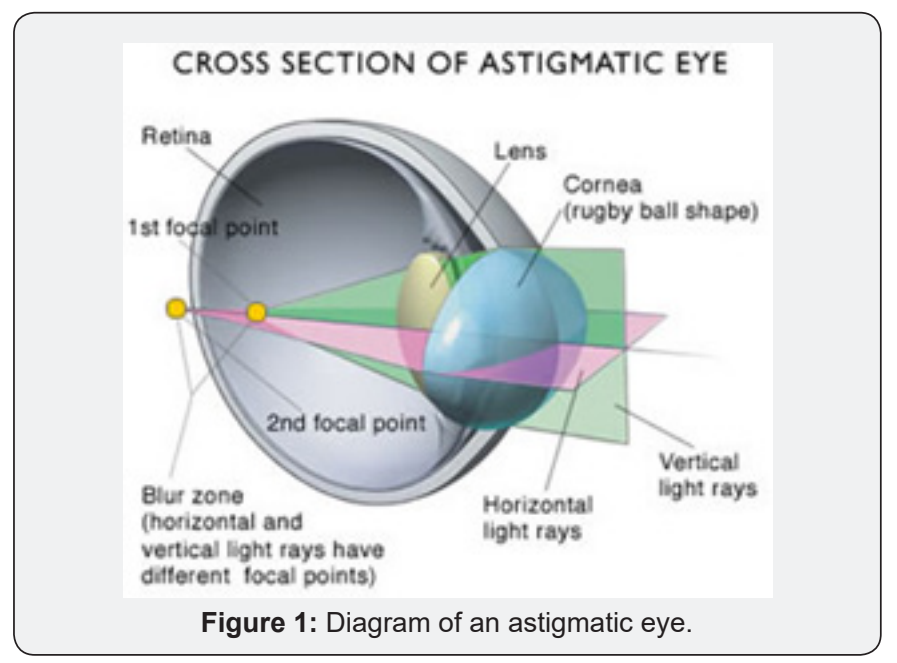

In an evolutionary study design of astigmatism to unfold characteristics changes with age, 3-4 years pre-school children were noted when they reached 8 years that hyperopic spherical equivalent and hyperopic astigmatism gradually reduces with age while myopic spherical equivalent increases with age [4]. Children who are highly astigmatic are not vulnerable to progress into high myopia [5]. According to some fanatics, lenticular cause of astigmatism is due to possible differences in refractive index or surface flattening rates of various lens' layers and /or asymmetry in layers that result in meridional difference [6]. Some further noticed that tilting or decentering of the lens induces astigmatism. Vitreous body suggestion came in that can also contribute by having different refractive index at interface between aqueous and hyaloid fossa. The summing up of all these is known as total astigmatism. It was scientifically well described by axes positions of cornea and lens were located. In some individuals it was noted that cornea can have irregular orientation of axes but overall astigmatism of globe being regular.

\section{Aetiology of astigmatism}

People at risk of having astigmatism are those with cornea ectasia like keratoconus, cornea scarring, high myopia or shortsightedness, high hyperopia or farsightedness, those who had ocular surgery or trauma. Some empirical reports were made that posterior globe surface pathology, staphyloma posticum which is characterized by soft axial elongation of globe causes irregular focal crests that can lead to astigmatism [7]. A detail was given that most astigmatism was observed in myopia as compared to hyperopia which hypothetically was in support that this was a directional dependent in correlation to posterior global distention.

Large lumps of the upper eyelids like chalazion on middle upper eyelid that compresses cornea can induce oblique stigmatism [8]. The corneal surface changes were also included. Explanation of pterygium which has invaded anterior cornea surface was studied [9]. It was seen that topographical pattern was altered and excision brought a reversal of manifested astigmatic effect. Iatrogenic causes were also summed up in which it was explained that cataract surgery wound closure with a suture material closer to the wound like visyn silk, monofilament $10 / 0$ were less irritating and also when placed near the wound allowed a good anatomical opposition [10]. The comparison of three suturing patterns using monofilament $10 / 0$ which bootlace which suture zig-zagged crisscrossed as shoe lace, single row suture zig-zagged without crisscrossed and limbal section which has interrupted sutures under conjunctival flap were seen to prevent sideways rotation of incision edges equally (Wishart, Gregor, 1986).

A comparison keratometry readings of incisions of anterior chamber tunnel of 6 and 10 millimeters from 4-millimeter length. It was evident that incision size altered corneal steepness by having $0.13 \mathrm{D}$ WTR in the first week of surgery which further increased to $0.22 \mathrm{D}$ in three months. Rapid wound stability had an advantage [11]. Another iatrogenic cause made an observation that dislocated Intraocular Lens (IOL) implant in the bag induced astigmatism. IOL repositioning led to myoic shift [12].

\section{Clinical features and epidermiology}

Epidemiological studies over the years have been indicating that astigmatism is found in $20-40 \%$ of the general population. It was also further surveyed that as little as $0.75 \mathrm{D}$ of astigmatism degrades images by causing haloes and ghosting of images as explained by Ghanem RC \& Azar TD [13]. Astigmatism was classified according to orientation of its angles. With rule astigmatism (WTR) has got the weakest meridian +/- 20 degrees between 160 and 20 degrees, against the rule Astigmatism (ATR) weakest meridian +/- 20 degrees between 110 and 60 degrees and oblique has got it between 110 and 60 degrees [14]. Among these axes' orientations, it was noted that higher spherical powers were common in WTR as compared to ATR and oblique stigmatism according to Rehvan et al. [15]. In young people between 3-5 years WTR was observed because of cornea contribution to optical system as noted by Shankar Sunita et al. [16]. It was further concluded that WTR was commonest in young subjects [6]. It explained that as age advances, axis orientation of total astigmatism from regular to irregular can happen because of horizontal cornea power increase and lenticular contribution. 
It is further classified according to refractive errors it presents with. If one of the images falls on the retina it is simple astigmatism. In accordance to where other image falls from the retina is further classified as simple myopic or hyperopic astigmatism. In one of the biggest retrospective study [17] of astigmatic corrections 51,000 patients were assessed. It analyzed that $67 \%$ of astigmatic corrections with $0.75 \mathrm{D}$ had a mirror symmetry within $10^{\circ}$ meaning that two axes added up to approximately $180^{\circ}$. Example if one is $165^{\circ}$ the other could be $15^{\circ}$ or one be $30^{\circ}$ other be $150^{\circ}$.

According to other observations [14], cases both images do not fall on retina and fall in front or behind it, it is called compound astigmatism. This is either known as compound hyperopic or compound myopic astigmatism. In other pattern is when images are not on retina, but one is in front and other is behind the retina it is known as mixed astigmatism.

\section{Signs and symptoms}

It was observed in an axis $15^{\circ}$ misalignment that is some individuals, develop a head tilt as a compensatory mechanism to improve vision [18]. This was also supported by El Nawawy in head, neck posture in compensation to vision condition in which eventually lead to predisposition chronic neuromuscular pain and musculoskeletal concern which eventually were also attending orthopedic surgeons' clinics (El Nawawy, 2012). Despite the postural challenges this was causing, Stolarz explained a physiological adaptation in a detail that tilting meridional acuity differences appreciated by astigmatic patients was corresponding to retinal responses not the spatial alignment to the stimuli. Cerebral cortex areas were not oriented in human constancy [19]. It was also quantitatively explained that this attempt to align eyes with visual environment was according to magnitude of astigmatic axis [20]. In regular astigmatism of more than $2 \mathrm{D}$ head tilt was noted to be at $5^{\circ}, 10^{\circ}, 15^{\circ}, 20^{\circ}$ and $25^{\circ}$. And it was concluded that axis orientation was not correlating with angle of compensatory head tilt.

In one survey of India among headaches study it was noted [21] that $65 \%$ of headaches were due to refractive errors. Breakdown of pattern refractive errors, astigmatism caused $41 \%$ cases followed by hyperopia $22 \%$ and myopia $12 \%$. In which it was concluded that ophthalmology plays a vital role in establishing and leading to a correct diagnosis of the medical problems associated with headache. The team of Albobashari, et al. [22] saw that the prevalence of chronic headache in ammetropia was higher than emmetropia subjects. It was also further seen that in their setting that $21 \%$ of headaches eventually were having an optometric consultation.

It was explained visual discomfort or asthenopia affects $40.8 \%$ day to day living more especially reading and doing near tasks association with temporal headache [23]. In the history taking it was explained photophobia, diplopia, tearing and blurriness of vision. Among all the refractive errors, astigmatism is noted to be the one most associated with aesthnopia with the highest peak to spectacle correction after 40 years when people begin to develop presbyopia [24]. In those who do a lot of close work on computers, astigmatic people were noted that those with uncorrected astigmatism develops early signs of computer vision syndrome and affects reading speed [25]. Some adapt by partially closuring eyelids which palpebral aperture is reduced to create an artificial stenopaeic slit permiting a horizontal meridian only to be focused.

\section{Diagnosis of astigmatism}

This requires a series of tests to be performed to subjects like astigmatic dial technique, stenopaeic slit, cross cylinder technique, Retinoscopy and others. Astigmatic dial technique is a procedure either plus or minus lens is presented to one eye at a time until Best Corrected Visual Acuity (BCVA) is obtained while one is occluded (Carlson Kurtz, 2014). Later two lines above BCVA are fogged by adding a plus lens. This makes a possible existing meridian to fall on the retina while the other one is pulled away. Then a patient looks at a chart (dial chart). Subject is asked if he or she notices whether both lines are equally blur or not. If patient sees 4 lines darkest, he or she has no astigmatism. It was also recommended that this was most dependable technique for refining amount of axis [26]. It was quantified that the lines which were 30 degrees apart were difficult to discriminate their axis locations. Also, had faced difficulties in neutralization of the principal meridians because appeared equally black.

Stenopaeic slit is a useful in examination of power and axis by the use of the rectangular aperture in an opaque black occluder [27]. Principle meridian is determined by reducing a blur circle. Axis orientation is noted by slow rotation of the slit. Eye fogging and defogging at 0.50 diopters steps helps to achieve best visual acuity. Then axis is placed $90^{\circ}$ from the previous axis and procedure is repeated. However, challenge that was being faced in this approach was that when it is not well oriented along one of the principal meridians according to geometrical optics, residual refractive error in a power space affect visual acuity [28].

Cross cylinder refraction technique is a high merited subjective technique which uses a lens with two cylindrical surfaces with equal radius. One is concave while the other is convex, and their axes cross at right angles [29]. Positions are marked with colours codes minus with white dot and plus with a red dot. It was named Jackson cross cylinder after its designer Edward Jackson in 1907. A contravening evidence that origin of a crossed cylinder was an astronomer, Airy [30]. Its handle is mounted at a 45 degrees angle to the cylinders. Technique gives BCVA by placing Circle of Least Confusion (COLC) at sturm's conoid creating mixed astigmatism but [31], a detail was added that JCC places the circle of least confusion at the outer limiting membrane of the retina as a method of probing presence of astigmatism. An arbitrary placement of cylinders 90 and 180 degrees during a flip tries to mark cylindrical locations in the peripheral visual field [32]. Gradual dialing to locate axis 
position is appreciated when using +/_ 0.25 when shifted at 22.4 degrees and $+/-0.50$ when shifted by 50 degrees [33].

For retinoscopy to be realized, Sir William Bowman reported perculiar pupillary reflex of astigmatic eyes in 1859 . First described by Cuigent in 1873. Later Copeland introduced streak retinoscope in 1973. Used are reflecting mirror and self-illuminated retinoscopy. In high astigmatism, the light streak deviates from the axis and this is called break and skew phenomenon. An examiner to be very certain about the reversal of the meridian reflexes separately. In theory it may be simple but in practice one has to subtract refraction from another amount. This was a recommendable cheaper alternative to help assessing those in his comparative study between normal and those with hearing impairment [34] used. This method was appreciated to be accurate method to assess refractive status objectively [35]. In a technologically advanced era was further quantified presentation in which a Plusoptix CR03 was compared to cycloplegic Retinoscopy outcome in which it was found average difference between the two was -0.77 with a p-value of 0.001 [36]. Elsewhere this was affirmed their research commende Plusoptix machine whose precision is closer retinoscopy to reliable equipment in addressing optical causes of amblyopia screening [37].

Reflex origin is believed to come from retina and vitreous junction. Large errors have dull reflex while small errors have a bright reflex. Reflex speed depends on residual ammetropia. The closeness the neutrality it is, the higher the speed. Broadens as it approaches the far point. If direction is with movement, meridian needs more plus and against it needs more minus. Neutral position happens when far point is conjugate with observer's nodal point.

\section{Current treatments}

It is corrected in three ways. These are refractive surgery, contact lenses and spectacle lenses. When using lenses astigmatism is corrected by different lenses. Initially it is done by spheres to correct the either myopia or hyperopia.

The divergence lenses are generally designed for myopia (shortsightedness). Myopia happens when eyeball is long or crystalline lens is too strong and light rays are bent too much that the focal point is in front of the retina. This makes the eye unable to see clearly things which are not far away. Glasses with convex lenses correct myopia by widening the plane of sight passing through the cornea that eventually pushes the focal point back towards the retina.

As for hyperopia this is opposite of myopia. The eye ball length could either be short or its crystalline lens been weak to bend light rays on the retina. This makes it difficult to see close things clearly. Glasses with convex lenses are used which narrows light plane as it passes through cornea and there is the resultant pulling of focal point towards the retina.
Once one meridian is neutralized then there is refining the focal point position of the second meridian precisely by a toric or cylindrical lens. These lenses are kind of divergent or convergent planes. This is done by the use of spherical lenses which either converges or disperses evenly.

The divergent lenses are placed parallel to a flatter meridian which eventually its convex shape gives a negative power against the steeper meridian which is perpendicular to it. This makes the image made by a steep meridian to move backwards and fall on the retina.

The convergent lenses are placed on top of a steep meridian which its effect will be on a flatter meridian which is oriented perpendicular to it. This makes the focal point made by a flat meridian to move forward and fall on the retina.

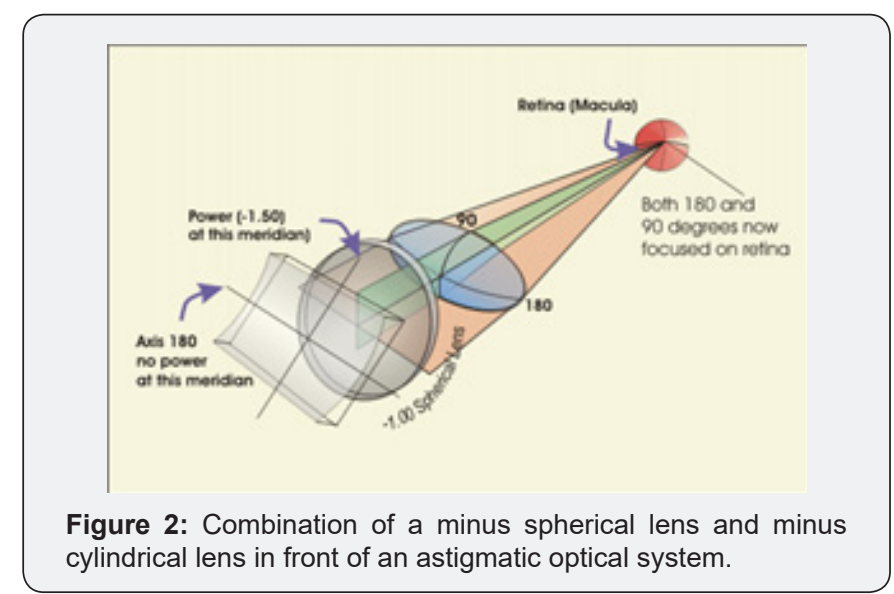

In general, these follow Knapp's law which explains that when a correcting lens is in front of an ametropic eye, when it's second principle plane is well coincided with the anterior focal plane, the resultant size of retinal image is similar to that of emmetropic eye [38] (Figure 2).

The overall toric lenses have got an invisible spoon shaped curve of its surface area that is placed directly perpendicular to the malformation of the eye ball which gives a perfect match to the unequal power meridians that makes the resultant viewing light annulus evenly rounded as it goes to the merge two focal points into one like a cone as being focused on the retina (Figure 3).

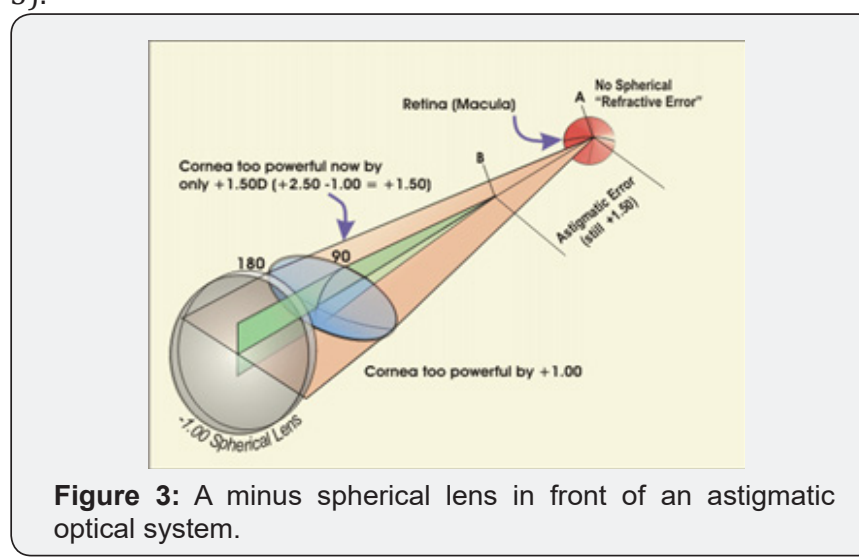


In some quarters as also explained the use of toric lenses has been ignored by using a full spherical power plus half of a cylindrical power disregarding the axis angle of astigmatism [38]. The best vision is achieved when the neck of circle of least confusion is located on the retina. A caution was made that its spherical equivalent should not exceed 1.00D [39]. It was further qualitatively observed according to the responses from spectacle wearers that image quality degrading somehow proportional to the increase of the Spherical Equivalent (S.E.) power. In qualitative observations SE's of $0.25 \mathrm{D}, 0.50 \mathrm{D}$ and 1.00 D its satisfactions were 43\%, 26\% and 19\% respectively.

The prescription [40]. The meridian's orientation is named counterclockwise in a range of 180 to 0 degrees facing the patient. This is documented as spherical power, cylindrical power and axis of a cylinder. The cylinder orientation is explained as a protractor placed in front of the eye with a degree marking as a reference.

In the view of the benefits of modern technological advancements, clinicians use contact lenses, refractive surgery or toric intraocular lens implants. But for some special reasons these might be resented elderly, patients with systemic and ocular contraindications or patients with ductylitis which can affect contact lenses insertion. Economic situation too can be a limitation factor for less industrialized setting for the counterparts to acquire and render the newer advanced technology and eventually rely only on spectacles as a means of refractive correction.

On the ways of treatment, an explore of treatment approach was made whether an equation can be used to predict a possible correlating spherocylindrical correction needed. The correlation coefficient between values and values of astigmatic eyes 0.991 [41].

\section{Objectives}

The study was aimed at finding the following:

a) Give an estimate of the difference in visual acuity outcomes of astigmatism eyes when corrected with spectacle cylindrical correction and spectacle spherical correction.

b) To show an existing land mark of spherical spectacle lenses that can give a clear image with an existing residual astigmatism in different cylindrical powers.

\section{Method and materials}

This study was carried out in Zomba, the former capital city of Malawi. The participants were from students from one secondary school teacher's college and learners from five secondary schools who provided informed consent prior to the study. They at first underwent a brief interview as guided by a data collection tool whether both or one eye had pertinent visual issues like ocular or systemic medications.

\section{Inclusion /Exclusion}

Inclusion criteria were all the patients who were more than 18 years old with astigmatism whose visual acuity was worse than $0.2 \log$ MAR and reached $0.0 \log$ MAR with cylindrical correction who were capable of giving a consent or minors, below 18 years whom authorities in their learning facilities gave a consent on their behalf as legal authenticity. Exclusion from this study were those who had ocular pathologies like media opacities, retinopathies or optic neuritis. All people who had pseudophakia bilaterally were excluded.

\section{Procedure}

All the examinations were made by four fully qualified optometrists and optometry technicians to ensure visual correction accuracy. A brief history was obtained as well as a meticulous and discerning physical examination was done using an ophthalmoscope in order to rule out presence of potential exclusionary criteria. A non-mydriatic fundoscopy was done to rule out posterior segment pathologies. Visual acuity of each eye was obtained using a Snellen visual acuity chart which was later converted into log MAR. This was followed by a subjective refraction which first was done by combination of spherical and cylindrical lenses then another visual acuity was obtained by the best sphere in each eye using a trial case and frame.

Reliability and efficiency of results was ensured by doing retesting in which was used to ensure degree of score reliability to come to 1 . Refraction was reproducible within $0.25 \mathrm{D}$ with minimal axis deviation within $1-3^{\circ}$. This accuracy was further ensured subjectively by avoiding amblyopia eyes and organic pathologies whose visual acuity drops could have caused inaccuracies. The use of competent optometrists to take part in the exercise ensured excellent measurements. All this ensured possibility of reproducibility of same results in healthy eyes.

Results

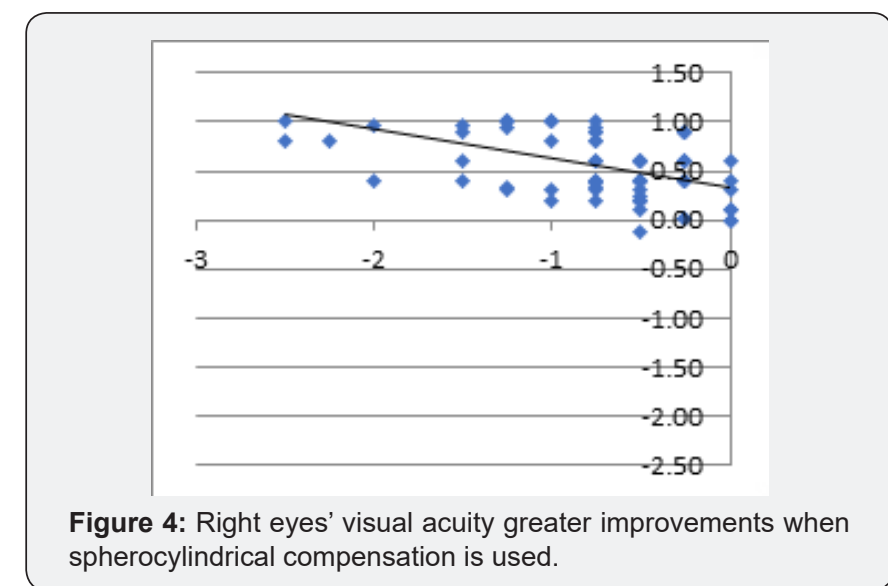

When analysing the results, it was found that both the visual acuity obtained with spherocylindrical correction and correction with spherical equivalent produced significant improvements in the VA of both eyes. RE mean and standard deviation of 
uncorrected VA is $0.5871 \pm 0.3692$. Spherocylindrical VA is $0.0251 \pm 0.0829$. VA with spherical equivalent is $0.3454 \pm 0.7476$; comparison between uncorrected VA and spherocylindrical VA: Z-score $=-6.2051, \mathrm{p}$ value $<0.001$. Comparison between uncorrected VA and VA with spherical equivalent: $\mathrm{Z}$ score $=$ -3.2394, p-value $=0.001$ (Figure $4 \& 5$ ).



Figure 5: Right eyes' less visual acuity improvement when spherical equivalent is used.

LE mean and standard deviation of uncorrected VA is 0.5637 \pm 0.3219 . Spherocylindrical VA is $0.0191 \pm 0.0707$. VA with spherical equivalent is $0.2340 \pm 0.2761$. Comparison between uncorrected VA and spherocylindrical VA: Z-score $=-6.6686$ p-value $<0.001$; comparison between uncorrected VA and VA of the spherical equivalent: Z-score $=-3.6185 \mathrm{But}$, as observed from previous data, spherocylindrical VA was significantly higher than the one obtained with the use of the spherical equivalent. This result was the same in both eyes (Figure $6 \& 7$ ).

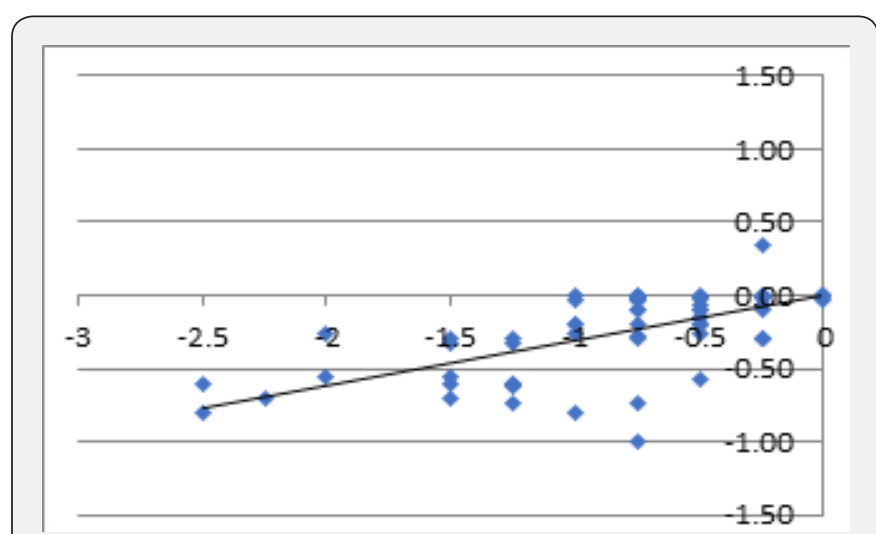

Figure 6: Left eyes' visual acuity greater improvements when spherocylindrical compensation is used.

RE comparison between spherocylindrical VA and VA with spherical equivalent: Z-score $=5.6403$ p-value $<0.001 ; \mathrm{LE}$ comparison between spherocylindrical VA and VA of the spherical equivalent: Z-score $=-5.6733 \mathrm{p}$-value $<0.001$. We calculate a regression line in which we observe the relationship between the cylindrical compensation of the patient and the difference between the VA obtained with spherocylindrical correction and the VA obtained with spherical equivalent. As it can be observed in Figures 3 \& 4, from 0 dioptres in RE and -0.125 dioptres in LE, there is a difference between the VA obtained by both methods of optical correction. This difference is greater as bigger is the cylindrical correction the patient needs.

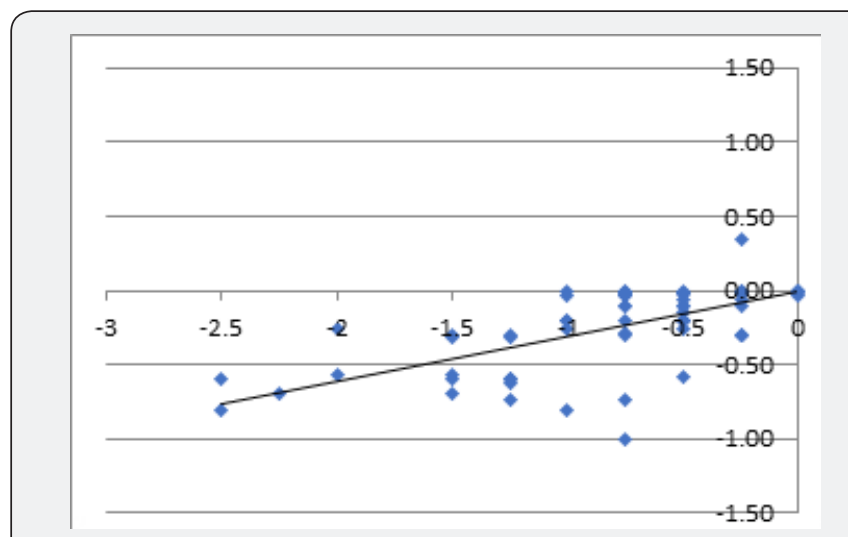

Figure 7: Left eyes' less visual acuity improvement when spherical equivalent is used.

Cycloplegic refraction is highly recommended in young subjects because they accommodate more and this cause discrepancies of $0.50 \mathrm{D}$ defined as pseudo myopia, with lead of accommodation noted in dynamic retinoscopy. As regards to availability of resources and time factor use of subjective refraction without cross checking with cycloplegia can be anticipated to cause a negligible concern of mean spherical equivalent error of $0.50 \mathrm{D}$ [42]. The noted in negligible differences in cycloplegic spherical equivalent and non-cycloplegic subjective spherical equivalents. In a similar complement noted that the comparison between spherical equivalent's subjective refraction and spherical equivalent's cycloplegic autorefraction being $+0.31 \pm 1.80 \mathrm{D}$ and $+0.31+0.61 \mathrm{D}$ respectively [42] .

In use of sophisticated equipment's, Grand Seiko WR 5100k, Retinomax and Canon RF10 in both either cycloplegic and noncycloplegic refraction it was noted that their refractive outcomes in subjects do differ somehow. It was also further noted that in abundance of such resources one has to work out quantification of actual results because all these had a phenomenon of overestimation resulting diagnosis of myopia in cycloplegia [43].

\section{Discussion}

The results show that both with spherocylindrical correction and correction with spherical equivalent there are improvements in VA. However, these improvements are greater with spherocylindrical correction. From 0 (RE) and -0.125 (LE) these differences can already be appreciated. From a statistical point of view, we should recommend the spherocylindrical correction from the lower astigmatism levels. Nevertheless, from a functional point of view, the optometrist can consider the possibility of using the spherical equivalent in the lowest cylindrical compensations.

The participants in this research were from Zomba, old capital city of Malawi which has a population of different social status dominated by rural and poor people who cannot afford 
purchasing appropriate spherocylindrical lenses correction. Furthermore, it is a district which has many learning institutions, national examination and teachers. It has been noted that many who seek glasses and teachers and scholars.

The results are very consistent with other existing publications from other researchers on the subject on the way gradual increment of spherical equivalent gives out a poor visual acuity. This study is important because it will help to overcome lack of precision while adjusting of spectacle prescription which is more suitable and affordable among individuals with astigmatism. It can be highly recommendable in subjects who are not, paediatric, amblyopic or going for refractive surgery. The data collector's accurate refraction skills subjectively without cycloplegics eliminated the costs, mydriatic drug's side effects, good handling of limited time allocated to this exercise which has enabled them to obtain three visual acuities from each stigmatic eye in a single refraction session. The use of cumbersome set of equipment, trial case, trial frame, Log MAR chart, writing materials and data collection tools enabled easy travelling to different places while trying to follow and draw subjects from different places to participate in the study [44-50].

The study's strength is minimising irrational use of analgesics that can result to drug dependency or addiction which to those struggling with asthenopia. Economically challenged people more especially living in rural areas will be capable of getting an alternative cheaper high-quality viewing glass. It can support proposal of protocols in economic challenges which can be part of ocular health integrated services in the future to minimise uncorrected refractive errors. This will help in situations where there is dispensation of already made spherical spectacle lenses to rural masses or when the optical practice has got limited supply of cylindrical lenses to be fitted. Trial frame and trial lenses multiple combinations was similar to spherocylindrical corrections and spherical equivalent corrections which subjects needed to obtain in their real-life setting.

The potential concerns of the model are that it will always require visual personnel to give the best decision in special astigmatism refractive state in paediatrics which poor correction can lead to disruption of visual functional development. This can happen when a child in critical stage of visual development switches from spherocylindrical correction to spherical equivalent correction. Another setback was lack of finances that could enable provision of glasses which eventually subjects could have been followed up on adaptation or presence of eyestrain over certain period of wearing. This is in contrary to some publications which had observed the subjects' extent of visual comfort in numerous ranges of spherical equivalent at specified time intervals as Reddy and colleagues did in India [39].

In this study comprised of simple and compound myopic eyes. In similar publication by Reddy it had shown that the vision reduction noted in either simple or compound astigmatism had similar trend [39]. The most important part which this study did not address is on possibility of cheaper technology or engraving of spherocylindrical power as an affordable power to those subjects who fail to have their visual acuity improved with spherical equivalent correction. Weaknesses noted in the study was that there was no spectacle lens provision to notice which patterns eventually will experience eye strain in spherical equivalent correction. It is also important to understand how spherical equivalent lens dispensation might affect binocularity of the eyes and stereopsis.

\section{Conclusion}

Through the noticing visual acuity changes between spherocylindrical lenses and spherical equivalent lenses simulation, it has been indicated that spherocylindrical correction gives the best visual acuity in all cylindrical powers whereas spherical equivalent gives less visual acuity improvements in high cylindrical powers. The spherical equivalent for spectacle lenses correction that will give high quality vision should be less than 1.00D.

\section{Annex 2: Data collection tool and consent form}

Date of examination:

\section{Introduction}

I am a student of School of Advanced Education, Research and Accreditation (SAERA) which is in Spain. I am studying for Master's Degree in Optometry and Vision Therapy. I am conducting a survey on the differences of visual acuity outcomes that can be achieved in a patient with astigmatism (or refractive errors which one eye has got two different refractive power meridians). Therefore, I request you to participate in this study honestly.

\section{Instructions}

a) All your eye examination will be kept confidentially; hence your name will not be written on this paper.

b) For those who are interested to know their number, confidentially will be given to them.

c) In this exercise you will benefit to know refractive powers that will be necessary to help each eye to see clearly.

d) The exercise will be short.

e) It will not be invasive that can cause a possible bodily harm or pain (Table 1-4).

CODE NUMBER OF A SUBJECT:

SEX: AGE:

OCCUPATION OR LEVEL OF EDUCATION:

OCULAR HISTORY: 
BRIEF MEDICAL HISTORY:

\begin{tabular}{|c|c|c|}
\hline Right Eye & & Left Eye \\
\hline & Visual Acuity without glasses & \\
\hline & Pinhole & \\
\hline & Near vision & \\
\hline & Eye lids & \\
\hline & Conjunctiva & \\
\hline & Cornea & \\
\hline & Anterior Chamber & \\
\hline & Iris & \\
\hline & Pupil & \\
\hline & Lens & \\
\hline & Extraocular muscles & \\
\hline
\end{tabular}

Posterior Segment

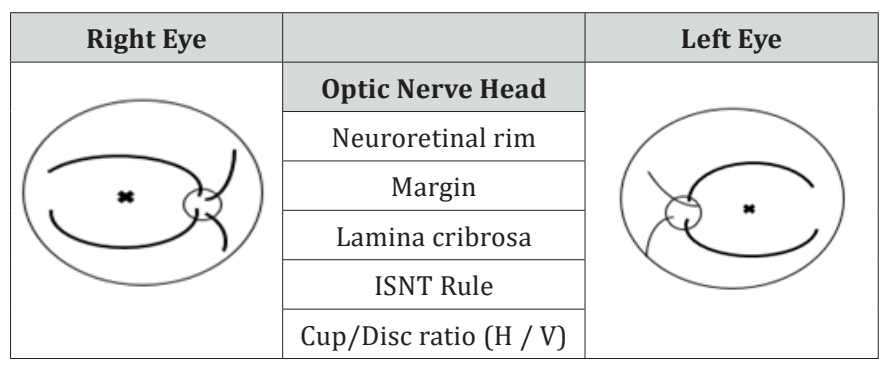

\begin{tabular}{|c|}
\hline Blood Vessels \\
\hline Colour Hue \\
\hline A.V. Ratio \\
\hline Pulse \\
\hline Macula \\
\hline Fovea Reflex \\
\hline Vitreous \\
\hline
\end{tabular}

Refraction

\begin{tabular}{|l|l|c|l|l|}
\hline & & Sphere / Cylinder x Axis - Visual Acuity & & \\
\hline & & Spherical Equivalent -Visual Acuity & & \\
\hline
\end{tabular}

\section{Annex 3: Letter requesting of educational institution authorities to collect data at the learning institution}

Ophthalmology Department,

Zomba Central Hospital,

P.O. Box 21 .

Zomba,

Malawi.

$27^{\text {th }}$ August 2018,

The Principal,
Dear Sir / Madam,

\section{REQUEST OF PERMIT TO CONDUCT DATA COLLECTION}

In partial fulfilment of requirement for Master's degree in Optometry and Vision Therapy 2018 thesis, I a student of School of Advanced Education, Research and Accreditation (SAERA) under University Isabel I Castella in Spain would like to ask for your permission to conduct a research study entitled "An experimental study of astigmatism optical correction on visual acuity changes when being corrected with spherocylindrical and spherical equivalent spectacle lenses".

In line with this I would like to ask your good office to allow me to do refraction and brief interview of your students in your vicinity. It will involve selection of refractive eye powers with two power meridians among those who wear spectacles or those with deranged vision willing to know their matching spectacle's power. Attach herewith is the copy of data questionnaire. Rest assured that the data I will gather will remain confidential and to the use of academic purpose only.

I believe that you are with me in enthusiasm to finish this requirement as compliance for my graduation and develop a well - being. I hope for your positive response on this humble matter.

Your approval to conduct this study will be greatly appreciated. Thank you.

Respectfully yours,

Innocent K. Thawe.

Optometry Student.

\section{Declaration}

I hereby declare that this submission is my own work towards master's degree in Optometry and Vision Therapy and to the best of my knowledge it contains no material previously published by another person nor material which have been accepted for the award of any degree of the university, except where due knowledge has been made to the text.

\section{Acknowledgements}

I owe a personal debt of gratitude to School of Advanced Education, Research and Accreditation (SAERA) and University Isabel I Castella for introducing this international master's degree programme for optometrists around the world. Thanks to the SAERA communications manager Ireen Namar from whom I first learnt more about the programme's details.

My thanks also go to the moderator Dr. Raủl Perez Ramos, my mentor Dr. Joaquin Vidal for the best guidance that has enabled me to come the good standard project. I sincerely appreciate the panel of lecturers who prepared and presented lessons so that they could be understood at an advanced level much better. 
I thank a lot my workmates and fellow optometrists who taught me the meaning of real friendship in helping data collection Messrs Chipiliro Chikapa, Samson Charles and Stanley Naphazi.

I also extend my profound gratitude to my brothers Martin and Patience Thawe for financing tuition to this learning tuition. Finally, I am not forgetting my mother who encouraged me a lot to study this programme and supported a lot in times of despair when confronted by challenges in this endeavor. I also do not take for granted those whom I have failed to mention their names individually who helped diversely to this work to appear a great one. May God bless you. I love you so much.

\section{Dedication}

In loving memory of my only sister, the late Tadala

\section{References}

1. Sorsby A, Sheridan M, Leary G (1962) Refraction and its components in twins. London: Medical Research Council 303(8): 1- 43.

2. Hammond CJ, Sneider H, Gilbert CE, Spector TD (2000) Genes and Environment in the refractive error: The twin eye study. Investigative Ophthalmology and Visual Science 42(6): 1232-1236.

3. Päraissinen O, Jauhonen H, Kauppinen M, Kaprio J, Konskevuo M, et al. (2013) Hereditary of spherical equivalent: a population - based twin study among 63 to 76-year-old female twins. Investigative Ophthalmology and Visual Science 54(9): 6063-6067.

4. Dobson V, Harvey EM, Miller JM (2007) Spherical equivalent refractive error in preschool children from a population with a high prevalence of astigmatism. Optometry and Vision Science 84(2): 124-130.

5. Harvey E, Twelker J, Miller J (2013) Relationship between refractive astigmatism and change in spherical equivalent with age in sample of native American children age 3 to 18 years of age. Investigative Ophthalmology and Visual Science 54(15): 2333.

6. Gündüz A, Evereklioglu C, Hepșen IF (2002) Lenticular astigmatism in tilted disc syndrome. Journal of Cataract and Refractive surgery 28(10): 1836-1840.

7. Thomson W (1875) Can staphyloma posticum induce astigmatism Transactions of the American Ophthalmological Society 79(2): 310318.

8. Jin KW, Shi YJ, Hyon JY (2017) Effects of chalazia on cornea astigmatism. BioMed Central Ophthalmology 17(1): 36.

9. Lindsay RG, Sullivan L (2001) Pterygium induced corneal astigmatism. Clinical and Experimental Optometry 84(4): 200-203.

10. Luntz MH, Livingston GL (1977) Astigmatism in cataract surgery. British Journal of Ophthalmology 61(5): 360-365.

11. Shepard JR (1989) Induced astigmatism in small incision cataract surgery. Journal of Cataract Refractive Surgery 15(1): 85-88.

12. Kristianslund O, Ølsten AE, Drosulm I (2017) Astigmatism and refractive outcome after a late in the bag intraocular lens dislocation surgery: randomized clinical trial. Investigative Ophthalmology and Visual Science 58(11): 4747-4753.

13. Ghanem RC, Azar TD (2008) Astigmatism and Cataract Surgery, In Jacobiec A and Miller A Principles and Practice in Ophthalmology Philadelphia: Saunders Elsevier 3(1): 1517.

14. Benjamin (2006) Borish Clinical Refraction. Oxford: Butterworth Heinemann, Elsevier.
15. Rehvan F, Yekta AA, Hashem H, Mehravan H, Ostadimoghadan $\mathrm{H}$, et al. (2011) The association between astigmatism and spherical refractive error in clinical population. Iranian Journal of Ophthalmology 23(4): 37- 42.

16. Sunita S, Bobier WR (2004) Corneal and lenticular components of total astigmatism in a preschool sample. Optometry and Vision Science 81(7): 536-542.

17. Solsona F (1975) Astigmatism as a congenital, bilateral symmetrical entity (observation based on the study of 51,000 patients. British Journal of Physiological Optics 30(2-4): 119-127.

18. Castro FA, Simao MI, Abbud CM, Foschini RM, Bicas HE (2005) Abnormal head position by incorrect prescription of astigmatism: Case report. Arquivos Brasileirs de Oftamologia, 68(5): 687-691.

19. Stolarz SJ (1975) The effect of head tilt on meridional differences in acuity: Implications for orientation constancy. Perception and Psychophysics 17(1): 17-22.

20. Ferashaki H, Azizzadeh A, Ghoreishi A, Fasihi M, Badiei M, et al. (2014) The effects of head tilt on ocular astigmatic axis, Advanced Biomedical Research 3: 10.

21. Jain S, Chandravanshi SL, Dukariya L, Tirkey ER, Jain SC (2015) Clinical study headache with special reference to ophthalmic cause. International Journal of Medical Science and Public Heath 4(2): 292-297.

22. Albobashari F, Hosseini SMA, Ali Yekta A, Khabazkhoob M (2014) The correlation between refractive errors and headache in young adults. Austin Journal of Clinical Ophthalmology 1(3): 1014.

23. Wajuihian SO (2015) Frequency of asthenopia with refractive errors. African Vision and Eye Health 74(1): 293-297.

24. Schellini S, Fabio F, Opromolla P, Oliviera L, Padovani C (2016) Main visual symptoms associated to refractive errors and spectacles need in Brazilian population. International Journal of Ophthalmology 9(11): 1657-1662.

25. Rosenfield M, Hue J, Huang R, Bababekova Y (2011) Uncorrected astigmatism and computer vision syndrome. Investigative Ophthalmology and Visual Science 52(14): 2815.

26. Regan JR (1937) Astigmatic dials in refined refraction. Archive Ophthalmolology 17(5): 788-796.

27. Choudhury PH, Shah BH (2018) Stenopeiac slit: A diagnostic tool. Ophthalmology Research: An international Journal 8(2): 1-3.

28. MunÄoz-EscrivaÂL and Furlan WD (2001) The Stenopaiec slit: an analytical expression to quantify its optical effects in front of astigmatic eye. Opthalmic and Physiological Optics 21(4): 327-333.

29. Harris WF (2007) Jackson Cross-Cylinder Part 1: Properties. The South African Optometrist 66(2): 41-55.

30. Friedman MD (1940) The Jackson crossed cylinder: A critique. Archive Ophthalmology 24(3): 490-499.

31. MunÄoz EscrivaÂ L, Furlan WD (2000) Jackson cross cylinder-simple formulation of its optical principles. Optica Applicata 30(2): 428-429.

32. Priors VD, Guyton DL (1986) A reappraisal, The Jackson Cross cylinder. American Academy of Ophthalmology 93(11): 1461-1465.

33. Elliot DB (2014) Clinical Procedures in Primary Eye Care. United Kingdom: Elsevier Saunders 4(10): 86.

34. Ostadimoghaddam H, Mirhajian H, Yekta AA, Rad DS, Haravian J, et al. (2015) Eye problems in children with hearing impairment. Journal of Current Ophthalmology 27(1-2): 56-59.

35. Duke Elder S (1950) Textbook of Ophthalmology, Vol IV London: Duke-Elder. 
36. Erdumus M, Yagci R, Karadag R, Durmus M (2007) A comparison of photorefraction and retinoscopy in children. Journal of American Association for Paediatric Ophthalmology 11(6): 606-611.

37. Moghaddam AAS, Kargozar A, Zarei Ghavanati M, Najjaran M, Nozari V, et al. (2011) Screening for amblyopia risk factors in preverbal children using the Plusoptix photoscreener: A cross sectional population - based study. British Journal of Ophthalmology 96(1): 83-86.

38. Wilkinson ME, Shadid KS (2017) Optics in Review. Iowa: Carver College of Medicine 1(19): 42.

39. Reddy BS, Das T, Mirda GS, Reddy N (2017) Patient Satisfaction and acceptance of spherical equivalent correction wear in rural India. Indian Journal of Ophthalmology 65(8): 729-732.

40. Schwartz SH (2013) Geometrical and visual optics New York: The McGraw Hill Companies 2: 129-130.

41. Moon B, Kim S, Cho H (2013) Predicting of uncorrected astigmatism from a decimal visual acuity from Decimal Visual Acuity in Spherical equivalent. Journal of Optical Society of Korea 17(2): 219.

42. Hashemi H, Khabazkhoob M, Asharlous A, Soroush, Yekta A, et al. (2016) Cycloplegic autorefraction versus subjective refraction: The Tehran Eye Study. British Medical Journal 100(8): 1122-1127.
43. Choong YF, Cheng A, Goh P (2006) A comparison of autorefraction with and without cycloplegia in primary school children. American Journal of Ophthalmology 142(1): 68-74.

44. Edgar S, Thomson MD (1908) Some clinical aspects of lenticular astigmatism. JAMA Ophthalmology 1(4): 302-307.

45. Elnawawy SMH (2012) Relationship between head and neck alignment and astigmatism (Unpublished Master's thesis) Cairo University.

46. Goldschmidt E, Jacobsen N (2014) Genetic end environmental effects of myopia development and progression. Eye 28(2): 126-133.

47. Hashemi H, Fatouchi A, Yekta A, Pkzad R, Ostadimoghaddam H, et al. (2017) Global and regional estimates of refractive errors: Systematic review and meta-analysis. Journal of Current Ophthalmology 30(1): 3-22.

48. Klein M (1944) Retinoscopy in Astigmatism. British Journal of Ophthalmology 28(5): 205-220.

49. Liang D, Guan Z, Lin J (1995) The relations of corneal, lenticular and total astigmatism. Chinese year 11(2): 70-72.

50. Tatham A, Pyrdal J (2008) Progressive lenticular astigmatism in clear lens. Journal of Cataract and Refractive Surgery 34(3): 514-516. 